\title{
miR-204 Shifts the Epithelial to Mesenchymal Transition in Concert with the Transcription Factors RUNX2, ETS1, and cMYB in Prostate Cancer Cell Line Model
}

\author{
Krassimira Todorova, Diana Zasheva, Kristiyan Kanev, and Soren Hayrabedyan \\ Institute of Biology and Immunology of Reproduction, Bulgarian Academy of Sciences, 73 Tsarigradsko Shosse Boulevard, \\ 1113 Sofia, Bulgaria \\ Correspondence should be addressed to Krassimira Todorova; krasiot@abv.bg
}

Received 29 June 2014; Revised 18 September 2014; Accepted 24 September 2014; Published 14 October 2014

Academic Editor: Vincent J. Gnanapragasam

Copyright (C) 2014 Krassimira Todorova et al. This is an open access article distributed under the Creative Commons Attribution License, which permits unrestricted use, distribution, and reproduction in any medium, provided the original work is properly cited.

\begin{abstract}
Epithelial to mesenchymal transition is an essential step in advanced cancer development. Many master transcription factors shift their expression to drive this process, while noncoding RNAs families like miR-200 are found to restrict it. In this study we investigated how the tumor suppressor miR-204 and several transcription factors modulate main markers of mesenchymal transformation like E- and N-cadherin, SLUG, VEGF, and SOX-9 in prostate cancer cell line model (LNCaP, PC3, VCaP, and NCIH660). We found that SLUG, E-cadherin, and N-cadherin are differentially modulated by miR-204, using miR-204 specific mimics and inhibitors and siRNA gene silencing (RUNX2, ETS-1, and cMYB). The genome perturbation associated TMPRSS2-ERG fusion coincided with shift from tumor-suppressor to tumor-promoting activity of this miRNA. The ability of miR-204 to suppress cancer cell viability and migration was lost in the fusion harboring cell lines. We found differential E-cadherin splicing corroborating to miR-204 modulatory effects. RUNX2, ETS1, and cMYB are involved in the regulation of E-cadherin, N-cadherin, and VEGFA expression. RUNX2 knockdown results in SOX9 downregulation, while ETS1 and cMYB silencing result in SOX9 upregulation in VCaP cells. Their expression was found to be also methylation dependent. Our study provides means for understanding cancer heterogeneity in regard to adapted therapeutic approaches development.
\end{abstract}

\section{Introduction}

Prostate cancer is one of the most common malignancies and the second leading cause of death from cancer in men. Androgen receptor (AR) is paramount for the lineage-specific differentiation of the prostate, inducing the expression of prostate-specific genes, such as PSA and TMPRSS2, and maintaining the differentiated prostate epithelial phenotype [1]. Cellular dedifferentiation and epithelial to mesenchymal transition (EMT), by contrast, are a hallmark of malignant transformation and metastatic disease. In this process the reexpression of conserved developmental programs plays a key role [2]. Chromosomal rearrangements fusing the androgen-regulated gene TMPRSS2 to the oncogenic ETS transcription factor ERG occur in approximately $50 \%$ of prostate cancers and more than $90 \%$ of them overexpress
ERG [3]. TMPRSS2-ERG is crucial for cancer progression by disrupting lineage-specific differentiation of the prostate and potentiating the histone methyltransferase EZH2-mediated dedifferentiation program. ERG disrupts AR signalling by inhibiting AR expression, binding to and inhibiting AR activity at gene-specific loci, and inducing repressive epigenetic programs via direct activation of the EZH2. The latter causes an epigenetic silencing of developmental regulators and tumor suppressor genes, subverting cancer cells to a stem-cell-like epigenetic state [4]. During this process, part of which is EMT, a transformation of epithelial cells into the invasive and proliferating mesenchymal cells occurs. The main event that follows is the transcriptional shift, involving ETS1, SLUG (SNAI2), and other transcription factors (TF) that suppress the epithelial markers (e.g., E-cadherin and $\beta$ catenin) and activate mesenchymal ones (e.g., $\mathrm{N}$-cadherin 
and vimentin), increasing the cell motility and cell migration [5]. Further under ERG control, even more invasive and proliferating phenotypes develop.

In $\mathrm{PCa}, \mathrm{AR}$ can function in two opposite directions: on one side, AR signalling is crucial for prostate and PCa cell survival; however its activation can also limit cell proliferation and mediate apoptotic induction under specific circumstances [6], like in conditions of genotoxic stress, when $\mathrm{AR}$ is fundamental for $\mathrm{p} 53$ activation and for the subsequent induction of apoptosis [7]. These modalities of AR depend to a large extent on the occurrence of the ERG fusion.

During this malignant progression phenomena of noncanonical binding of AR by transcriptional factors occur, which "modify" AR signalling in cancer prosurvival manner. Thus, the EMT involved TF ETS1 was shown to physically interact with AR [8] and its binding motif was found enriched in the AR binding sites. Similarly, ERG redirects AR to other genes that are not normally androgen stimulated, like SOX9. SOX9 was found crucial for normal prostate development and also for prostate cancer initiation and further advancement [2]. Recent studies confirmed SOX9 as a critical downstream effector of ERG. It mediates androgen dependent prostate morphogenesis and AR mediated prostate cancer development. Sox 9 is associated with increased cell invasiveness in VCaP.

Recently, another transcription factor RUNX2 has been implicated as the one driving bone marrow metastatic homing of prostate cancer cells. In bone marrow, these cells compete for the hematopoietic stem cells niche, a process that is RUNX2 dependent. Normally, RUNX2 stimulates osteoblast differentiation of mesenchymal stem cells [9].

On the other hand, the hematopoetic lineage differentiation is dependent on another transcription factor that has been recently implicated in cancer metastasis, the protooncogene c-MYB $[10,11]$.

MicroRNAs (miRs) are noncoding RNAs that exert a role of posttranscriptional regulators, silencing specific mRNA targets by binding specific seed sequences at their $3^{\prime}$ UTR $[12,13]$. Two major events determine the noncoding RNAs as another major player in malignant progression. First, the genome rearrangement results in gain-of-function and loss-of-function events due to the generation of new or the deletion of existing microRNA seed regions. MicroRNAs sequences could be altered as well. Second, AR mediates miR maturation in Dicer [14] in ligand dependent manner, participating in three-step pathway including miR activation, AR corepressor suppression, and DNA interaction to elicit its action. Loss of DICER results in androgen-insensitivity syndrome. miRs are mediators of $\mathrm{AR}$ function and the existence of a possible feedback loop between miRs, AR, and AR corepressors [14]. This implicates them directly in prostate carcinogenesis and progression as a mechanistic factor determining AR signalling specificity in androgen independent prostate cancers. It is not surprising that some novel prostate cancer specific markers, like AMACR [15] and PSMA $[16,17]$, were also found dependent on different micro RNAs [18].

The miR-200 family was found to inhibit the epithelialmesenchymal transition and cancer cell migration by direct targeting some of E-cadherin transcriptional repressors [19]. Member of the same family, miR-204 has been found to target RUNX2 in mice [20] and to be highly expressed in normal renal tissue. The promoter of the gene harboring miR-204 was found to contain an ETS1 binding site [29].

We investigated how miR-204 and the master TFs (SLUG, SOX9, RUNX2, ETS1, and cMYB) are implicated in EMT (Ecadherin, $\mathrm{N}$-cadherin) and in the expression of angiogenesis marker VEGFA, using cell lines model LNCaP [21], PC3 [22], $\mathrm{VCaP}$ [23], and NCI-H660 [24] of AR sensitive and refractive prostate cancer cell lines, being either TMPRSS2-ERG fusion harboring or fusion-free.

\section{Materials and Methods}

2.1. Cell Lines. LNCaP (lymph-node metastasis-derived), androgen receptor $(\mathrm{AR})$ responsive $(\mathrm{AR}+)$, p53 enabled; $\mathrm{PC} 3$ (bone metastasis derived, p53 null and AR-unresponsive $(\mathrm{AR}-)$ ); $\mathrm{VCaP}$ (vertebral bone metastasis derived, $\mathrm{AR}+$ ); and NCI-H660 (lymph-node prostate small cell carcinoma, AR-) prostate cancer cell lines were purchased from the ATCC (VA, US). LNCaP cells were grown in RPMI media (ATCC, Catalog number 30-2001), PC3 were grown in ATCC-formulated F-12 K Medium (Catalog number 30-2004), and VCaP cells were grown in DMEM media (ATCC Catalog number 302002) supplemented with $10 \%$ fetal bovine serum (Sigma, St. Louis, MO, USA). The H660 cells were grown in RPMI media (ATCC, Catalog number 30-2001, complete growth media contain $0.005 \mathrm{mg} / \mathrm{mL}$ insulin, $0.01 \mathrm{mg} / \mathrm{mL}$ transferrin, $30 \mathrm{nM}$ sodium selenite (final conc.), $10 \mathrm{nM}$ hydrocortisone (final conc.), $10 \mathrm{nM}$ beta-estradiol (final conc.), extra $2 \mathrm{mM}$ L-glutamine (for final conc. of $4 \mathrm{mM}$ ), $5 \%$ fetal bovine serum (final conc.) (Sigma, St. Louis, MO, USA)). All cell lines were cultivated in a humidified atmosphere at $5 \% \mathrm{CO}_{2}$ and $37^{\circ} \mathrm{C}$.

2.2. MicroRNA Transfection. The miR-204 inhibitor (antihsa-miR204), mimic (syn-hsa-miR204), and their respective negative controls (MiScript Inhibitor Negative control and AllStars Negative siRNA) (Qiagen, Hilden, Germany) were transfected into $\mathrm{LNCaP}, \mathrm{PC} 3, \mathrm{VCaP}$, and $\mathrm{H} 660$ cells by HiPerFect (Qiagen, Hilden, Germany) for $24 \mathrm{~h}$, according to the manufacturer's instruction. The cells were seeded a day before transfection in 24-well plate (TPP, Trasadingen, Switzerland) for RT-qPCR, in 12-well plate (TPP, Trasadingen, Switzerland) for cell migration assay and flow cytometry assay, and in 96-well plate (TPP, Trasadingen, Switzerland) for cell proliferation assay (CCK-8). Experiments were done in triplicate.

2.3. Cell Viability Assay. The cell viability assay was done for cells transfected with syn-hsa-miR204, AllStars Negative siRNA, anti-hsa-miR204, or MiScript Inhibitor negative control in 96 -well plate format $(100 \mu \mathrm{L}$ cell suspension volume/well). The measurement was done using the Cell Counting Kit- (CCK-) 8 (Sigma-Aldrich, St. Louis, MO) assay, which is based on the conversion of water-soluble tetrazolium salt, WST- 8 to a water-soluble formazan dye upon reduction in the presence of an electron carrier by 
dehydrogenases [25]. The assay was performed by adding $10 \mu \mathrm{L}$ CCK- 8 to each well for $4 \mathrm{~h}$ at $37^{\circ} \mathrm{C}$. The OD was read at $450 \mathrm{~nm}$ using a multiplate reader Flowstar OPTIMA (BMG LabTech, Germany). Experiments were done in triplicate.

2.4. Cell Migration Assay. Cell migration assay was done for cells transfected with syn-hsa-miR204, AllStars Negative siRNA, anti-hsa-miR204, or MiScript Inhibitor negative control in 12-well plate format. LNCaP, PC3, and VCaP cells were seeded a day before transfection with $80 \%$ confluence. For this assay we used a p200 pipet tip to create a scratch of the cell monolayer after transfection. The plate was washed and replaced with cultivation medium. An invert Leica light microscope equipped with digital camera was used for time lapse study. The examination of the cell migration was done on 0, 6, 12 and 18 hours. Experiments were done in triplicate. Using ImageJ software we measured the distance traveled by the cells during the time points and calculated the relative change in the scratch width measured in arbitrary units for each treatment.

2.5. syn-hsa-miR204 and Anti-hsa-miR204 Efficiency Validated Using miR-204 Specific Luciferase Reporter Assay. LNCaP, PC3, VCaP, and H660 cell lines were seeded in a 96-well plate (Orange, Braine-l'Alleud, Belgium) and transfected for $24 \mathrm{~h}$ with Negative siRNA, miR-204 mimic, and miR204-inhibitor. All wells were transfected with $5 \mu \mathrm{g} 3^{\prime} \mathrm{UTR}$ GoClone luciferase reporter construct containing $3^{\prime}$ UTR seed specific for miR-204 (Product ID S880168) at the end of Luciferase encoding gene. The reporter is optimized specifically for use with the LightSwitch Luciferase Assay System (SwitchGear Genomics, Carlsbad, CA, USA), and has basal high Luciferase signal, which is reduced upon miR-204 specific binding. All transfections were done with HiPerfect (Qiagen, Hilden, Germany). Experiments were performed in triplicate. After adding the assay solution to wells and 30 min incubation at RT in the dark, the plates were read in a FLOWStar Optima reader (BMG LABTECH GMBH, Ortenberg, Germany).

The reporter data was analyzed by estimating the ratio of the signal obtained by miR-204 mimic or other specific treatment and the signal obtained by nontargeted microRNA. Qiagen control resembling the latter is MiScript Inhibitor Control (Qiagen negative control for miR inhibitors, binds only AllStars Negative Control siRNA). The estimated ratios for miR-204 mimic and miR-204 inhibitor were further normalized to the reporter data for AllStars Negative Control siRNA (Qiagen control for miR mimics, same size as miRs, optimized for minimized off-target effects) and reported in arbitrary units of Luciferase reduction of microRNA mimic/inhibitor compared to nontargeting control microRNA like sequence.

2.6. RUNX2, ETS1, and cMYB Knockdown with Small Interfering RNA (siRNA). For siRNA knockdown of RUNX2, ETS1, and c-MYB, we used human mission esiRNAs (Sigma, St. Louis, MO, USA) against the target molecules with the following design:
RUNX2: GGTACCAGATGGGACTGTGGTTACTGTCATGGCGGGTAACGATGAAAATTATTCTGCT

\section{ETS1: TGAGACCTTCCAAGGACAGCCGTGTTG-} GTTGGACTCTGAATTTTGAATTGTTATTCTAT

cMYB: GGGCAGTAGAGCTTGGACAGAAAGAAAAGAAACTTGGTGGTAGGTAATTGACTATGCA.

AllStars Negative control siRNA $(5 \mathrm{nmol})$ and AllStars HsCell Death Control siRNA ( $5 \mathrm{nmol}$ ) were used as a control of transfection (Qiagen, Hilden, Germany). LNCaP, PC3, $\mathrm{VCaP}$, and $\mathrm{H} 660$ cells were seeded a day before transfection with $80 \%$ confluence in a 24 -well plate (TPP, Trasadingen, Switzerland) for RT-qPCR. Transfections were done according to the manufacturer's instruction with HiPerFect (Qiagen, Hilden, Germany). $200 \mathrm{nM}$ of siRNA was used for transfection for $72 \mathrm{~h}$. Experiments were done in triplicate. siRNAs knockdown of RUNX2, ETS1, and $C M Y B$ was validated by RTqPCR and flow cytometry assays.

2.7. Real-Time Reverse Transcription Quantitative PCR Analysis. The expression of two sets of genes-set 1: SLUG, Ecadherin, N-cadherin, VEGFA, and SOX9; set2: RUNX2, ETS1, and $c M Y B$-was detected by RT-qPCR. After $24 \mathrm{~h}$ mimic or inhibitor and $72 \mathrm{~h}$ esiRNAs transfection mRNA was isolated from LNCaP, PC3, VCaP, and H660 cells using Midiprep kit (Qiagen, Hilden, Germany). From each sample 500 ng total RNA was used to synthesize cDNA by Sensiscript Reverse Transcription kit (Qiagen, Hilden, Germany). Firststrand cDNA synthesis was performed according to the manufacturer's instruction (Qiagen, Hilden, Germany) and 500 ng cDNA was used for PCR reactions (SYBR Green QuantiTect RT-PCR MasterMix, Qiagen, Hilden, Germany). Total reaction volume was $50 \mu \mathrm{L}$. Primer concentrations were $0.5 \mu \mathrm{M}$ (Fw and Re). RT-PCR Cycler (Agilent Technologies MX3005P, Stratagene, Santa Clara, CA, USA) was used in this study. PCR reactions were started at $95^{\circ} \mathrm{C}$ for $15 \mathrm{sec}$, then $50^{\circ} \mathrm{C}$ for $30 \mathrm{sec}, 72^{\circ} \mathrm{C}$ for $30 \mathrm{sec}$, followed by 45 cycles. The mRNA transcript expression levels of all studied genes were normalized towards transcript levels of endogenous reference gene phosphoglycerate kinase 1 (PGK1). PGK1 is a transferase enzyme that has been considered as a housekeeping gene for the cells. PGK is found in all living organisms and its sequence has been highly conserved throughout evolution [26].

The following primer sequences, designed by us and produced by Biomers (Ulm, Germany), were used:

SLUG Fw: $5^{\prime}$-ata ttc gga aca cat tac- $3^{\prime}$, Re: $5^{\prime}$-gca aat gct ctg ttg cag tga- $3^{\prime}$;

E-cadherin (CDH1) Fw: $5^{\prime}$-aga acg cat tgc cac ata cac tc- $3^{\prime}$, Re: $5^{\prime}$-act gca ttc ccg ttg gat gac ac- $3^{\prime}$;

$N$-cadherin (CDH2) Fw: $5^{\prime}$-tgg gaa tcc gag gaa tgg- $3^{\prime}$, Re: $5^{\prime}$-tgc aga tcg gac cgg ata ct- $3^{\prime}$;

VEGFA Fw: $5^{\prime}$-cct tgc tgc tct acc tcc ac- $3^{\prime}$, Re: $5^{\prime}$-cca tga act tca cca ctt cg- $3^{\prime}$;

SOX9 Fw: $5^{\prime}$-gag gaa gtc ggt gaa gaa cg- $3^{\prime}$, Re: $5^{\prime}$-atc gaa ggt ctc gat gtt gg- $3^{\prime}$;

PGK1 Fw: $5^{\prime}$-att agc cga gcc agc caa aat ag- $3^{\prime}$, Re: $5^{\prime}$-tca tca aaa acc cac cag cct- $3^{\prime}$; 
MYB Fw: $5^{\prime}$-aag tct gga aag cgt cac ttg- $3^{\prime}$, Re: $5^{\prime}$-aca tct gtt cga ttc ggg aga ta- $3^{\prime}$;

RUNX2 Fw: $5^{\prime}-\operatorname{cgg} \operatorname{ccc}$ tcc ctg aac tct- $3^{\prime}$, Re: $5^{\prime}-\operatorname{tcg} \operatorname{ctg} c c t$ ggg gtc tgt a-3';

ETS1 Fw: $5^{\prime}$-agt gct caa gga cat cga gac g- $3^{\prime}$, Re: $5^{\prime}$-agc cac ttc tgc aca ttg $\operatorname{ctg}-3^{\prime}$.

2.8. Flow Cytometry Validation of siRNA Knockdown. Flow cytometry was used instead of western blotting as protein detection method following an already established approach $[27,28]$. Transfected (negative siRNAs and esiRNAs: RUNX2, ETS1, and cMYB) prostate cancer cells (LNCaP, PC3, VCaP, and $\mathrm{H} 660$ ) were detached with Accutase (eBioscience, Frankfurt, Germany) and washed with cold $1 \%$ BSA-PBS. Specific antibodies (Abs) (Santa Cruz Biotechnology, Dallas, Texas, USA) were used for detection of RUNX2, ETS1, and cMYB: RUNX2 $(27-\mathrm{K})$ is a mouse monoclonal antibody raised against recombinant RUNX2 of human origin; ETS1 (C-4) is a mouse monoclonal antibody raised against amino acids 131280 of ETS1 of human origin; c-Myb (D-7) is a mouse monoclonal antibody raised against amino acids 500-640 of c-Myb of human origin. After IC Fixation/Permeabilization solution (eBioscience, Frankfurt, Germany) wash and flow cytometry staining buffer (eBioscience, Frankfurt, Germany), the specific primary Abs or the appropriate isotype control Abs were used at concentration of $0.5 \mu \mathrm{g} / 106$ cells for $60 \mathrm{~min}$ on ice, followed by BSA-PBS wash and secondary antibody (rabbit anti-mouse FITC conjugated IgG, Santa Cruz Biotechnology, Dallas, Texas, USA) incubation at $0.25 \mu \mathrm{g} / 106$ cells for $30 \mathrm{~min}$ on ice (in the dark). Cells were gated using forward versus side scatter to exclude dead cells and debris. After washing, cells were analyzed with a BD FACSCalibur flow cytometer (Becton Dickenson, Franklin Lakes, NJ, USA). Fluorescence of $10^{4}$ cells per sample was acquired in logarithmic mode for visual inspection of the distributions and for quantifying the expression of the relevant molecules by calculating the median fluorescence intensity (referred to as MFI) in histogram overlay graphics.

2.9. Total Demethylation. LNCaP, PC3, VCaP, and H660 cells were seeded in a 24-well plate (TPP, Trasadingen, Switzerland) for RT-qPCR. The cells were treated with $6 \mu \mathrm{M}$ 5-Azacytidine (Sigma, St. Louis, MO, USA) (5-AzaC) for four days. The media were changed every day with fresh 5-AzaC solution. Briefly, total RNA was isolated from the cells and cDNA was synthesized following the same protocol described above. RT-qPCR was performed to measure the expression of SLUG, E-cadherin, N-cadherin, VEGFA, and SOX9 genes. Experiments were done in triplicate.

2.10. Statistical Analysis. The data were generated from three independent experiments, each performed in triplicate. One-way ANOVA test with respective multiple comparison posttests (Greenhouse-Geisser correction for nondata sphericity, Tukey correction posttest, adjusted $P$ value, and family-wise significance, confidence level of 0.05 ) was used to analyze the data (GraphPad Prism 6, La Jolla, CA, USA). $P<0.05$ was considered significant.

\section{Results and Discussion}

3.1. E-Cadherin and N-Cadherin Are Differentially Affected by miR-204 Overexpression Depending on the Level of Genome Perturbation of the Prostate Cancer Cell Line Model. The miR-204 was found only slightly expressed in intact human prostate tissue [29], but overexpressed in PC3 and DU145 prostate cancer cell lines [30]. While it is reported as tumor suppressor in some cancers [31,32], its role in prostate cancer is rather oncogenic [30]. Due to the limited amount of studies and the dualistic behavior of miR-204, we decided to follow the miR-204 modulatory effect on molecules important for EMT such as SLUG, E-cadherin, and N-cadherin.

We induced miR-204 overexpression using synthetic mimics and miR-204 suppression using specific inhibitor and validated their functional efficiency using Luciferase reporter bearing miR-204 specific seed region (Figure 1).

We found that E-cadherin was upregulated in LNCaP cells and downregulated in all other cell lines, while $\mathrm{N}$ cadherin was upregulated in all four cell lines after miR204 mimic transfection. SLUG was upregulated only in VCaP cells (Figure 1). VCaP and NCI-H660 cell lines harbor the TMPRSS2-ERG fusion and have an advanced genome perturbation status. The downregulation in NCI-H660 was stronger than in other cell lines (Figure 1), and it could be explained with additional E-cadherin alternative splicing exon-11 exclusion, associated with prostate and other malignant tumors [33]. E-Cadherin is downregulated, while Ncadherin is upregulated during EMT [5].

3.2. E-Cadherin Differential Regulation by miR-204 in LNCaP Cells Probably Depends on Its Different Splicing Variant Switch. We found additionally that E-cadherin is alternatively spliced in LNCaP and all other cell lines: PC3, VCaP, and NCIH660. This was manifested by a different melting curve peak when E-cadherin was amplified in either LNCaP and PC3 or other two cell lines (Figure 2). This alternative splicing might account for the E-cadherin downregulation during cancer progression and metastasis and could further support the differential miR-204 suppressive effect exerted in prostate cancer cell lines discussed above.

This could be explained by the different events of alternative isoform expression like alterations in tandem $3^{\prime}$ untranslated regions that correlate to the different cancer phenotypes [34].

3.3. miR-204 Upregulates SOX9 and Regulates VEGFA in an AR Sensitivity Dependent Manner in Prostate Cancer Cell Line Model. SOX-9 was upregulated by miR-204 in all cell lines, while SOX9 downregulation by miR-204 inhibition was lost in the fusion harboring cell lines (Figure 1). The importance of miR-204 implication in Sox9 control is related to the finding of the role of Sox 9 as imperative for both early prostate development and prostate cancer (TRAMP and Hi-Myc) initiation as demonstrated by studies utilizing its deletion. This is due to its role in multiple cytokeratins and cell adherence/polarity regulation [2]. Moreover, SOX9 is found as critical downstream effector of ERG in TMPRSS2:ERG 


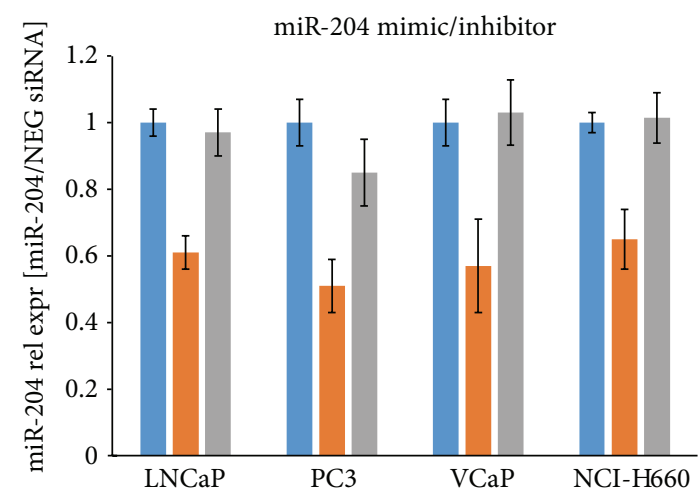

- NEG siRNA

- miR-204 mimic

- miR-204 inhibitor
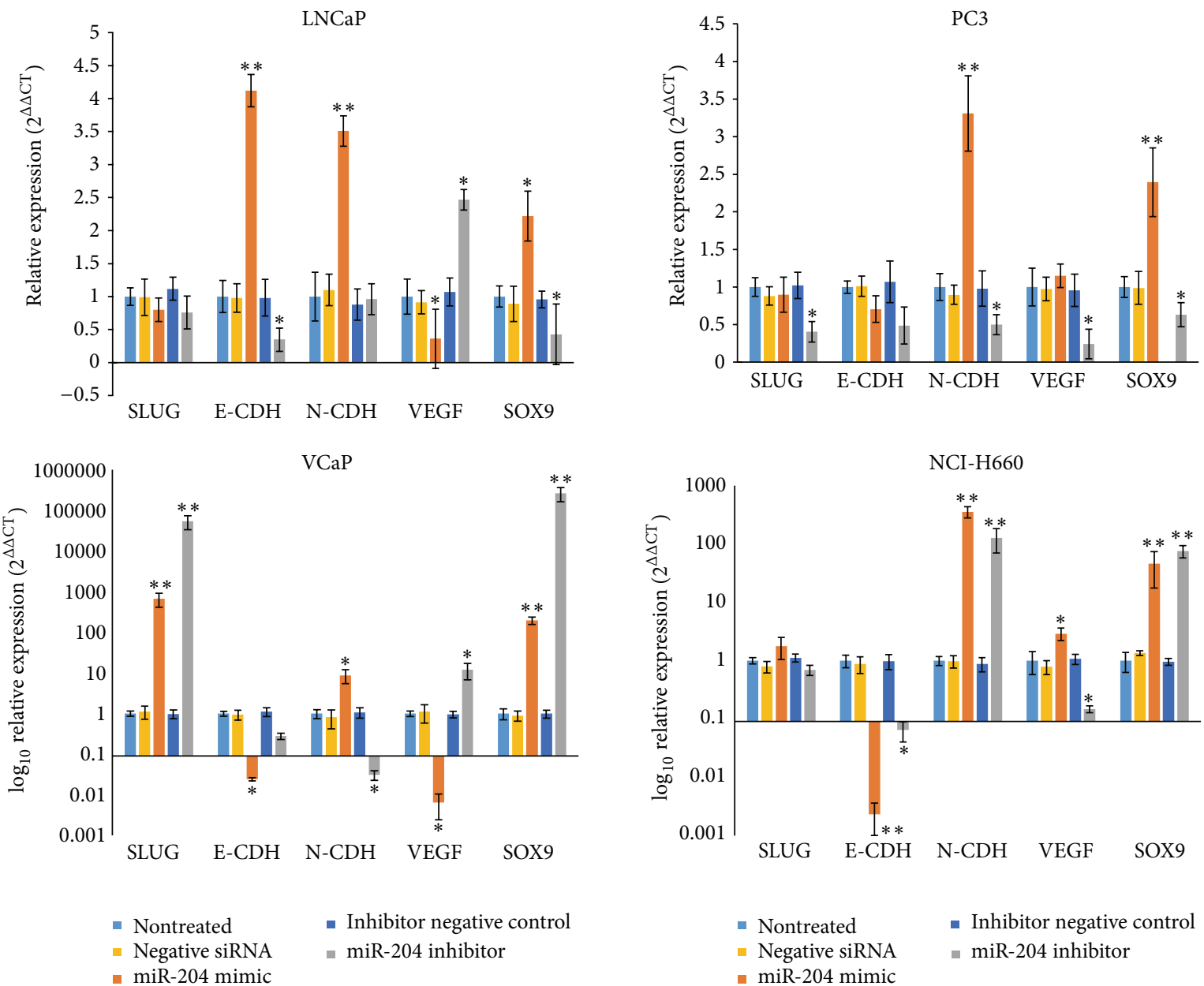

FIGURE 1: Luciferase reporter assay validation of syn-hsa-miR204 and anti-hsa-miR204 efficiency. Luminescence readings (arbitrary units) of miR-204 seed specific LightSwitch 3'UTR GoClone Luciferase reporter cotransfected with MiSrcipt Inhibitor Negative Control (targeting only AllStars Negative siRNA) and miR-204 mimic, or miR-204 inhibitor, normalized to AllStars Negative siRNA cotransfection. miR-204 mimic binding to the reporter $3^{\prime}$ UTR results in decreased Luciferase signal. Effect of miR-204 on EMT. AllStars Negative siRNA, MiSrcipt Inhibitor Negative Control, miR-204 mimic, or miR-204 inhibitor transfected LNCaP, PC3, VCaP, and NCI-H660 cell lines were harvested for total RNA. qPCR was performed for SLUG, ECDH, NCDH, VEGFA, SOX9, and the relative gene expression (Pfaffl, $2^{\triangle \triangle C T}$ ), normalized to $P G K 1$, was assessed for LNCaP, PC3, VCaP, and NCI-H660 cell lines. ${ }^{* *} P<0.01,{ }^{*} P<0.05$. Error bars represent s.d., $n=3$ independent biological repeats. 


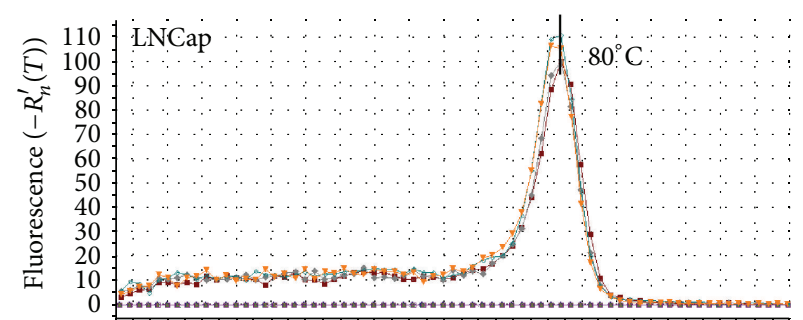

5658606264666870727476788082848688909294 Temperature $\left({ }^{\circ} \mathrm{C}\right)$
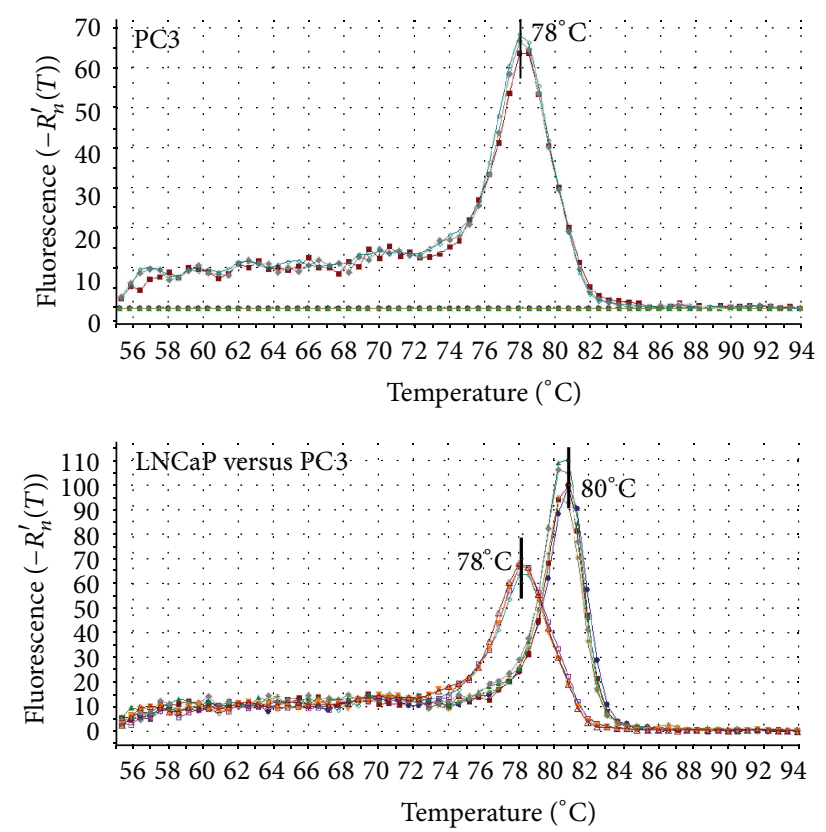
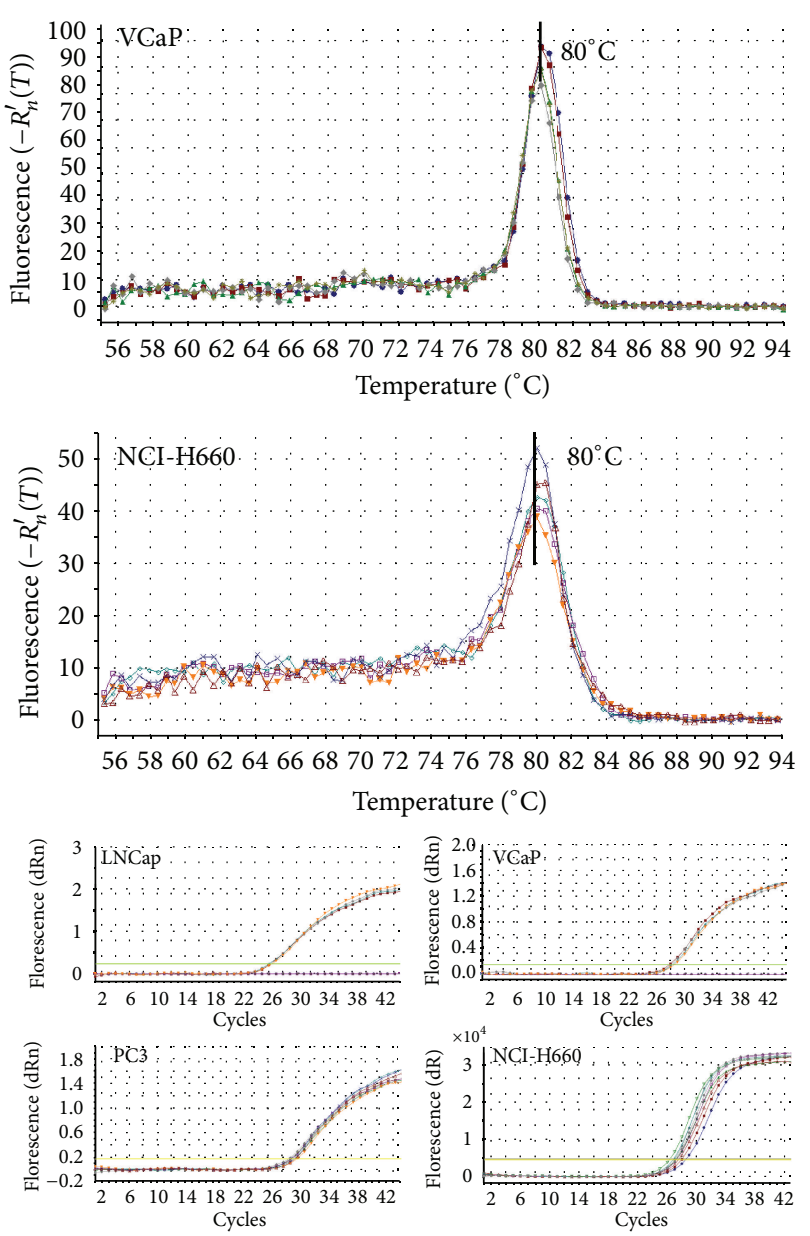

FIGURE 2: E-Cadherin has differential splicing variant expression in LNCaP cells compared to all other cell lines: PC3, VCaP, and NCI-H660. Dissociation and amplification curves (Stratagene MX3005P) of ECDH specific primers amplified product in total RNA from LNCaP, PC3, VCaP, and NCI-H660 cells (AllStars Negative siRNA, MiSrcipt Inhibitor Negative Control, miR-204 mimic, or miR-204 inhibitor). Different Tm in dissociation curves are highlighted using vertical black bars.

fusion-positive PCa like VCaP. Since ERG is overexpressed in these fusion cells, and ERG redirects AR to a set of genes including SOX9 that are not normally androgen stimulated. This further supports miR-204 deregulation role in cancer progression [35].

We followed the role of miR-204 in VEGFA transcript expression since many metastases secrete VEGFA to produce neovascularization [36]. miR-204 modulated VEGFA in ARsensitivity dependent manner. It downregulated VEGFA in LNCaP and VCaP cells and upregulated VEGFA in castration resistant cell lines as PC3 and NCI-H660. The inhibition of miR-204 had a reciprocal effect on VEGFA transcripts (Figure 1). This miR is known to regulate the vascularization in cornea [37] by inhibiting Angiopoetin-1 and also to play substantial role in pulmonary arterial hypertonia [38], where it is downregulated.

3.4. miR-204 Suppresses Cell Viability and Migration Only in TMPRSS2:ERG Fusion-Negative Cell Lines. Using MTT assay we found that miR-204 mimic decreased cell viability in LNCaP and PC3 cells, while it had no significant effect in both
TMPRSS2:ERG fusion-positive cell lines VCaP and NCIH660 (Figure 3). miR-204 inhibitor produced an opposite, positive effect on cell viability, but we found it significantly increased only VCaP cells (Figure 3). Similar to that observation, the cell migration was decreased by miR-204 mimic in LNCaP and PC3 cells, while in VCaP cells the mimic had no significant effect (Figure 4). miR-204 inhibitor increased cell migration in all three cell lines (Figure 4).

3.5. RUNX2, ETS1, and cMYB Are Required for SLUG Expression in TMPRSS2-ERG Free Cell Lines LNCaP and PC3. SLUG is in vivo recruited by the promoters of Runx 2 and Sox 9 genes, thus acting as both positive and negative transcription regulator of these genes, respectively [39]. We followed how the transcription factors RUNX2, ETS1, and cMYB were involved in prostate cancer related EMT.

Runx2, a bone-specific transcription regulator, is abnormally expressed in highly metastatic prostate cancer cells, promoting metastasis, driving the metastatic homing to the bone marrow hematopoietic stem cell niche, and facilitating local osteolysis [40]. This is achieved through large tumors 

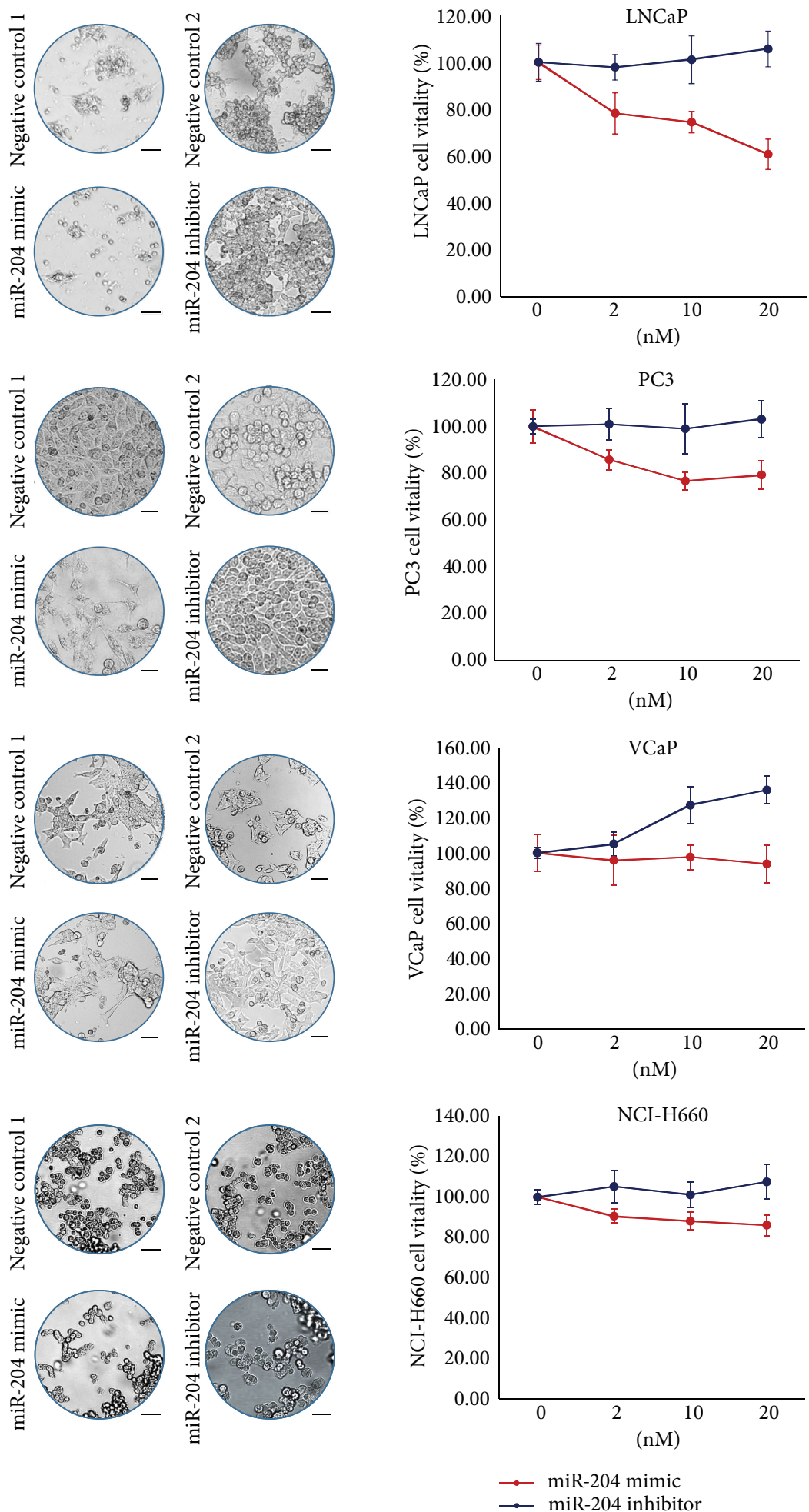

FIgURE 3: miR-204 decreases LNCaP and PC3 cell viability but has no effect on VCaP and NCI-H660 viability. Changes in cell viability of LNCaP, PC3, VCaP, and NCI-H660 cells transfected for $24 \mathrm{~h}$ with either AllStars Negative siRNA, MiSrcipt Inhibitor Negative Control, miR204 mimic, or miR-204 inhibitor in increasing dosages $(2,10,20 \mathrm{nM})$ were assessed using cell viability kit CCK-8. Colorimetric assay was read on $450 \mathrm{~nm}$. Scale bar corresponds to $50 \mu \mathrm{m} .{ }^{* *} P<0.01,{ }^{*} P<0.05$. Error bars represent s.d., $n=3$ independent biological repeats. 


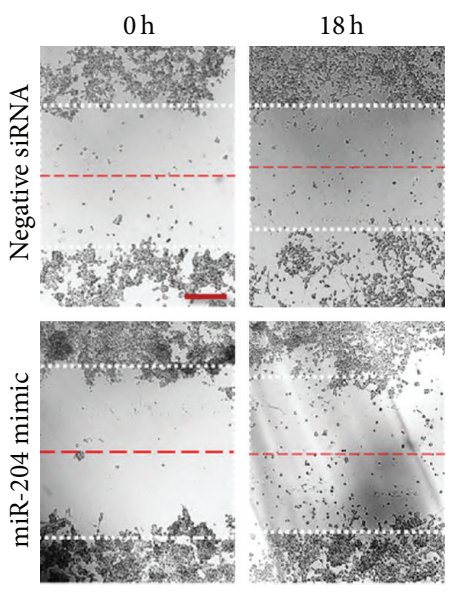

$0 \mathrm{~h}$
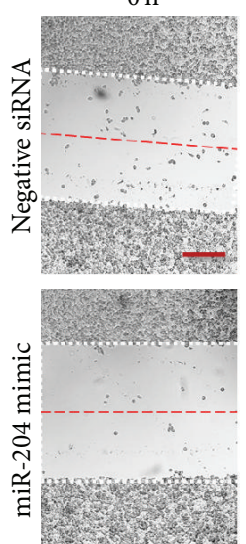

$0 \mathrm{~h}$

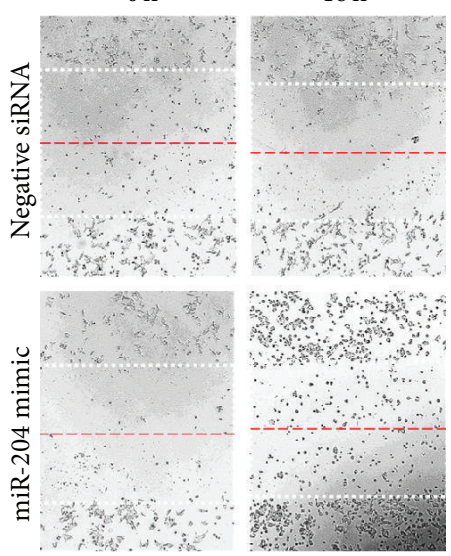

$0 \mathrm{~h}$

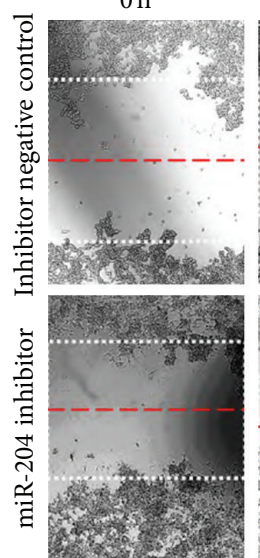

$0 \mathrm{~h}$

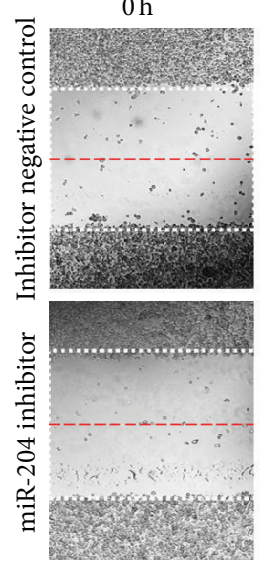

$0 \mathrm{~h}$

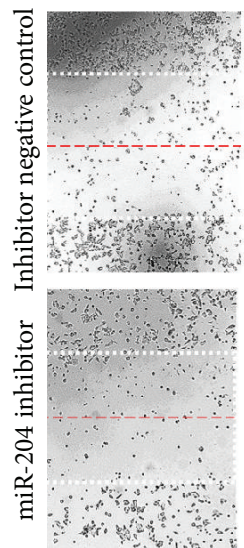

$18 \mathrm{~h}$

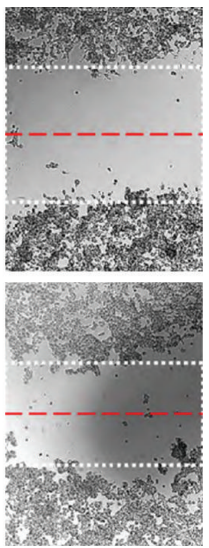

$18 \mathrm{~h}$

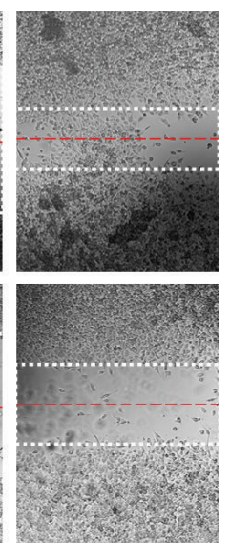

$18 \mathrm{~h}$

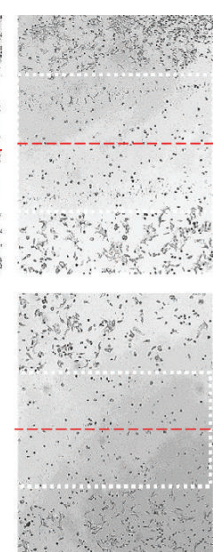

Migration assay, LNCaP

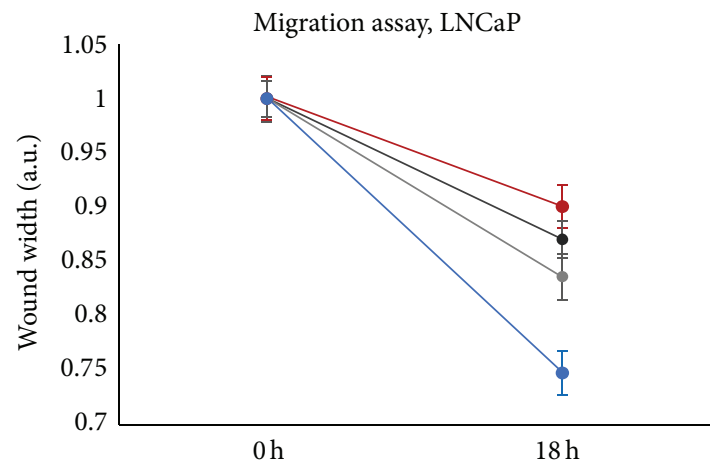

Migration assay, PC3
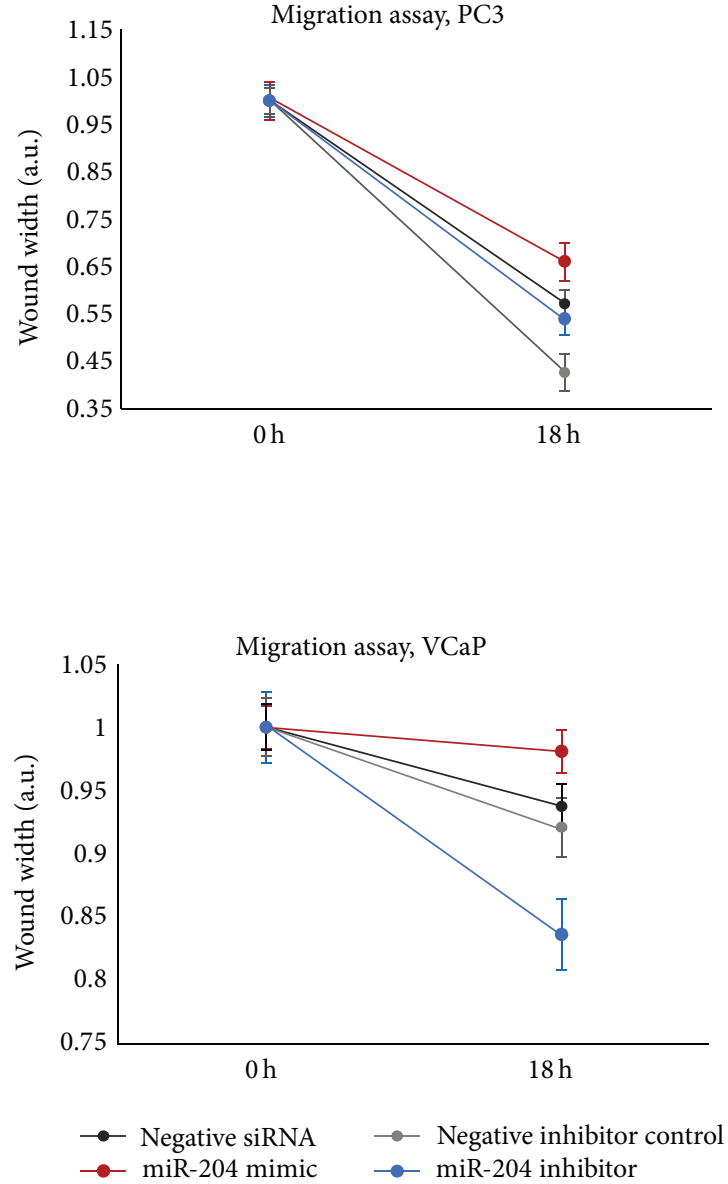

FIGURE 4: miR-204 suppresses cell migration in TMPRSS2:ERG fusion-negative cell lines. Cell migration assay was done for cells transfected with syn-hsa-miR204, AllStars Negative siRNA, anti-hsa-miR204, or MiScript Inhibitor negative control. Morphometric measurement of the scratch width at $0 \mathrm{~h}$ and $18 \mathrm{~h}$ time points, represented as relative change in arbitrary units for each treatment. Error bars represent s.d., $n=3$ independent biological repeats.

development, increased expression of matrix metalloproteinases (MMP9, MMP13), osteomineralization inhibition (Osteopontin), bone-resorbing factors (PTHrP, IL8), and VEGF, a key angiogenic factor [36, 40]. Key event to this process is the induction of EMT-related molecules (SOX9,
Snail2/SLUG/SNAI2, and SMAD3) by RUNX2 that potentially enhance invasion of cancer cells $[36,41]$.

As SLUG is reported to upregulate RUNX2 [42], we decided to follow the reverse pathway of interaction, namely, if RUNX2 affects SLUG levels. Our results showed that SLUG 

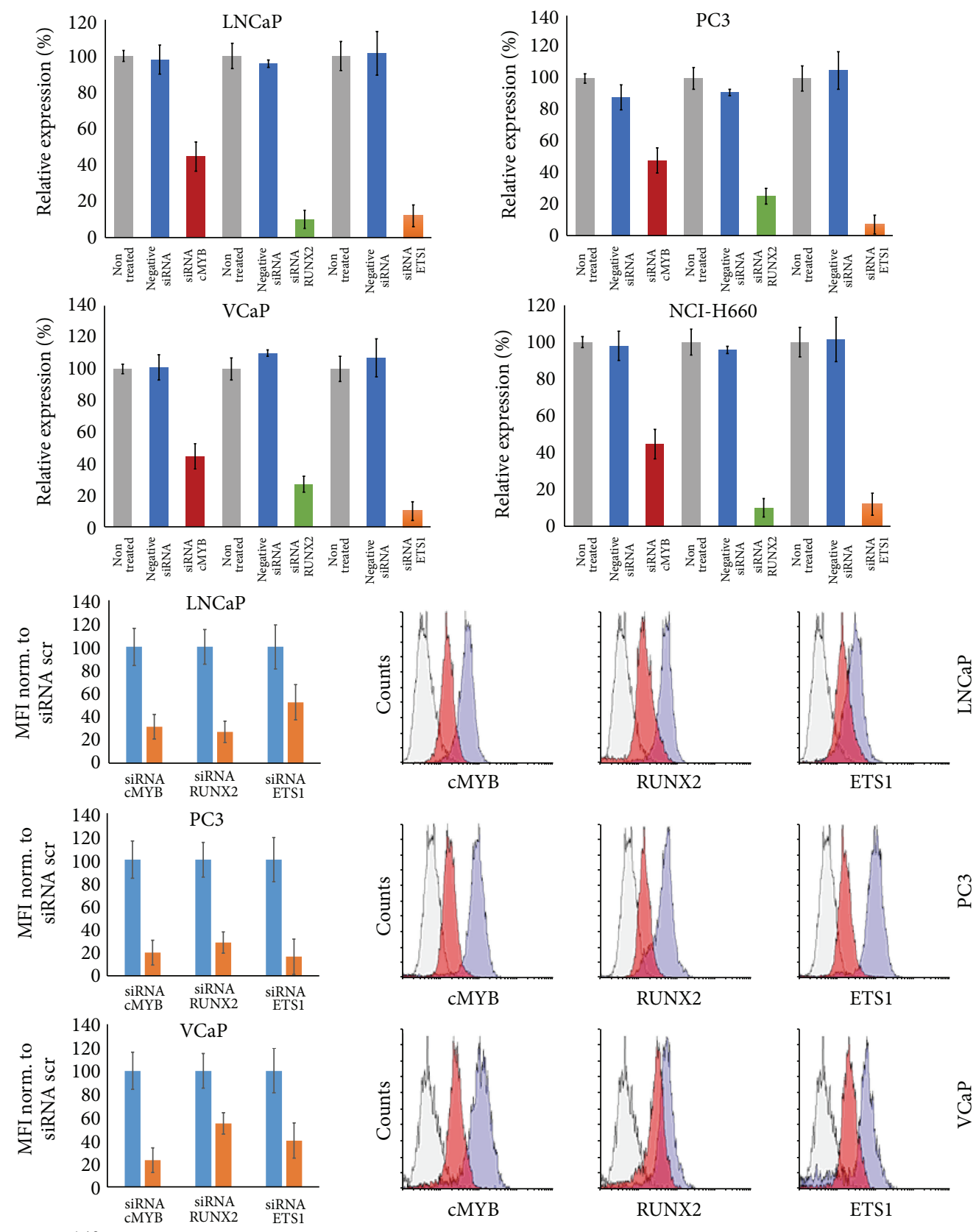

RUNX2

ETS1

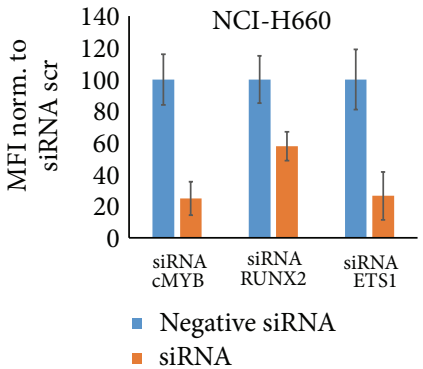

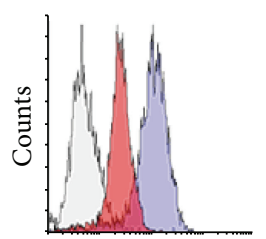

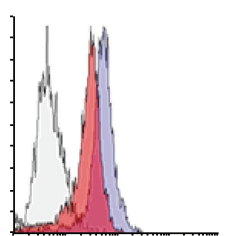

RUNX2

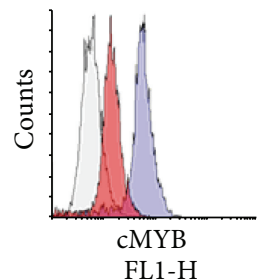

$\square$ Isotype control

$\square$ Negative siRNA contro

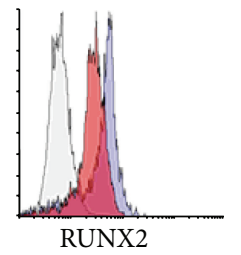

FL1-H

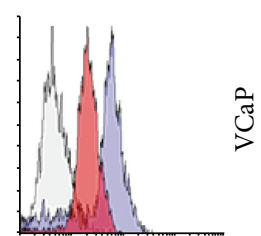

ETS1

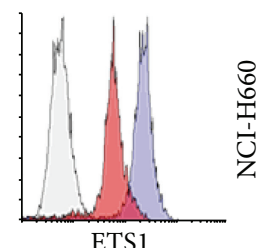

ETS1

FL1-H

$\stackrel{3}{3}$

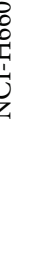

Figure 5: Transcription factors cMYB, RUNX2, and ETS1 siRNA knockdown validation. qPCR validation. Cell lines LNCaP, PC3, VCaP, and NCI H660 were analyzed for cMYB, RUNX2, and ETS1 mRNA expression by RT-qPCR after specific siRNA or AllStars Negative siRNA transfection. qPCR data are represented as a relative fold change normalized to nontreated cells (100\%). FCS validation. Cell lines LNCaP, PC3, VCaP, and NCI H660 were analyzed for cMYB, RUNX2, and ETS1 protein expression after specific siRNA or AllStars Negative siRNA transfection using FCS. Expression is presented as FCS overlay histograms of isotype control, AllStars Negative siRNA, and specific siRNA transfected cells. MFI bar chart represents the mean fluorescent intensity index of each FCS overlay histogram, normalized to MFI of AllStars Negative siRNA transfected cells (100\%). 

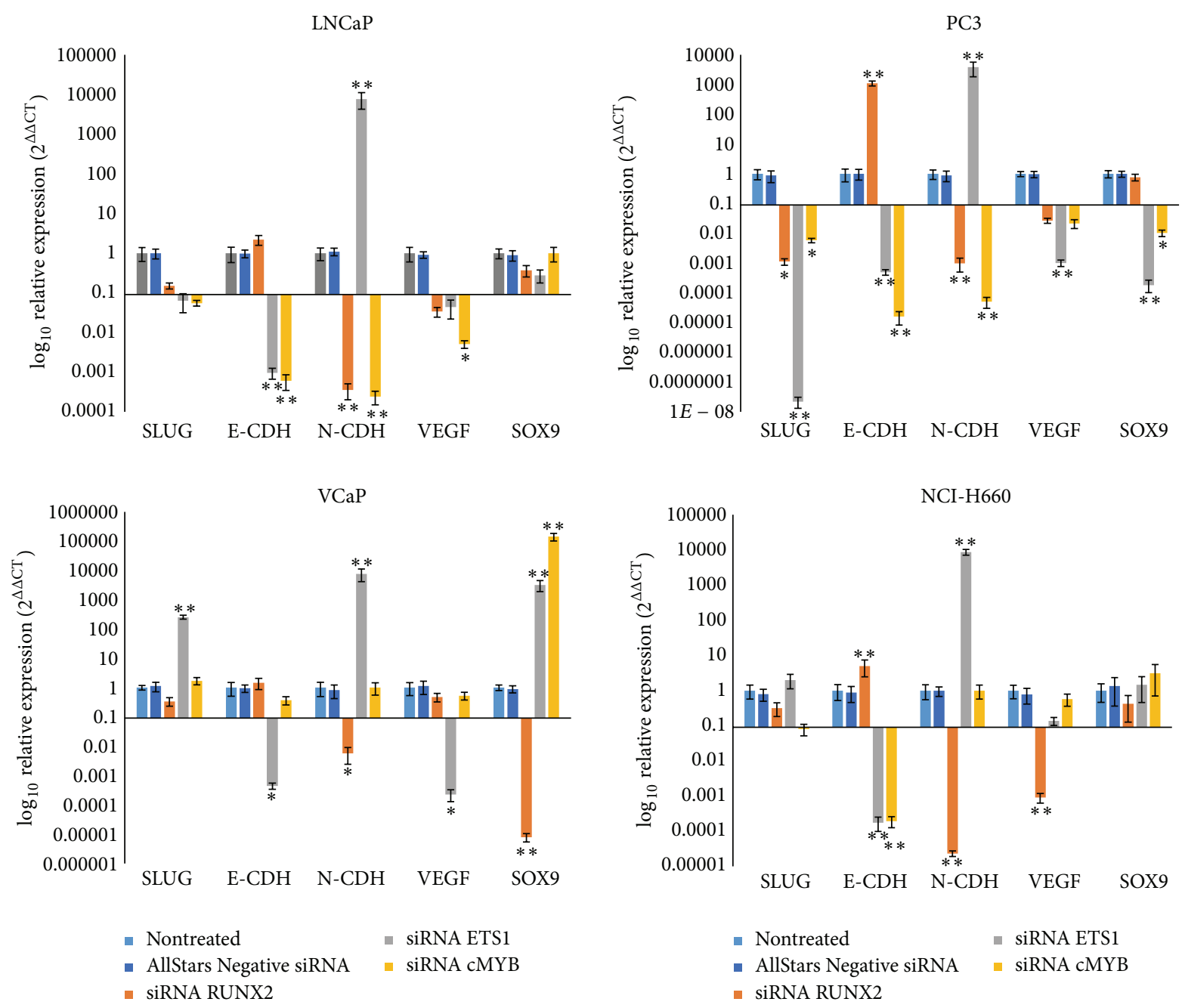

FIGURE 6: Transcription factors cMYB, RUNX2, and ETS1 are EMT regulators. LNCaP, PC3, VCaP, and NCI-H660 cells transfected for $72 \mathrm{~h}$ using either AllStars Negative siRNA or siRNA specific for RUNX2, ETS1, or cMYB, were all harvested for total RNA. qPCR was performed for SLUG, ECDH, NCDH, VEGFA, SOX9, and the relative gene expression represented as $\log _{10}$ change (Pfaffl, $2^{\Delta \Delta C T}$ ), normalized to PGK1, was assessed for LNCaP, PC3, VCaP, and NCI-H660 cell lines. ${ }^{* *} P<0.01,{ }^{*} P<0.05$. Error bars represent s.d., $n=3$ independent biological repeats.

was downregulated after RUNX2 silencing in all four cell lines (Figures 5 and 6), suggesting that there is a positive feedback loop between RUNX2 and SLUG. Indeed, recent data show that RUNX2 is able to bind AR and detach it from its target genes like some tumor suppressors, but at the same time, the two together, RUNX2 and AR, are able to bind the SLUG enhancer region and result in an increased invasiveness [43].

ETS1 silencing resulted in SLUG downregulation in both LNCaP and PC3 cells, while there was no significant effect in NCI H660 cells. Surprisingly, in VCaP cells ETS1 silencing resulted in SLUG upregulation (Figures 5 and 6). cMYB silencing resulted in SLUG downregulation in all lines, but $\mathrm{VCaP}$, where it had no significant effect (Figures 5 and 6).

3.6. RUNX2, ETS1, and cMYB Are Involved in the Regulation of E-Cadherin, $N$-Cadherin, and VEGFA Expression. Silencing ETS1, we found it to be a positive E-cadherin and a negative $\mathrm{N}$-cadherin regulator, accordingly, thus preventing EMT. Silencing of RUNX2 resulted in downregulation of $\mathrm{N}$-cadherin in all cell lines. Interestingly, it resulted in concomitant upregulation of E-cadherin in PC3 and NCI H660 AR irresponsive cell lines, but not in AR sensitive LNCaP and VCaP cell lines (Figures 5 and 6).

In noncancer mouse mammary cells, Ets1 was found to facilitate TGF- $\beta$ induced EMT and E-cadherin downregulation specifically, by upregulation of direct E-cadherin transcription repressors [44]. Our data show an inverted ETS1 action in human prostate cancer, most likely driven by the androgen and TMPRSS2:ERG fusion signaling.

cMYB silencing revealed that it is also a positive Ecadherin regulator, except in $\mathrm{VCaP}$ cells. We found that cMYB is required for EMT in LNCaP and PC3 cells, as cMYB silencing downregulated $\mathrm{N}$-cadherin. This dependence was lost in TMPRSS2-ERG fusion harboring cells like VCaP and NCI-H660 (Figures 5 and 6). 

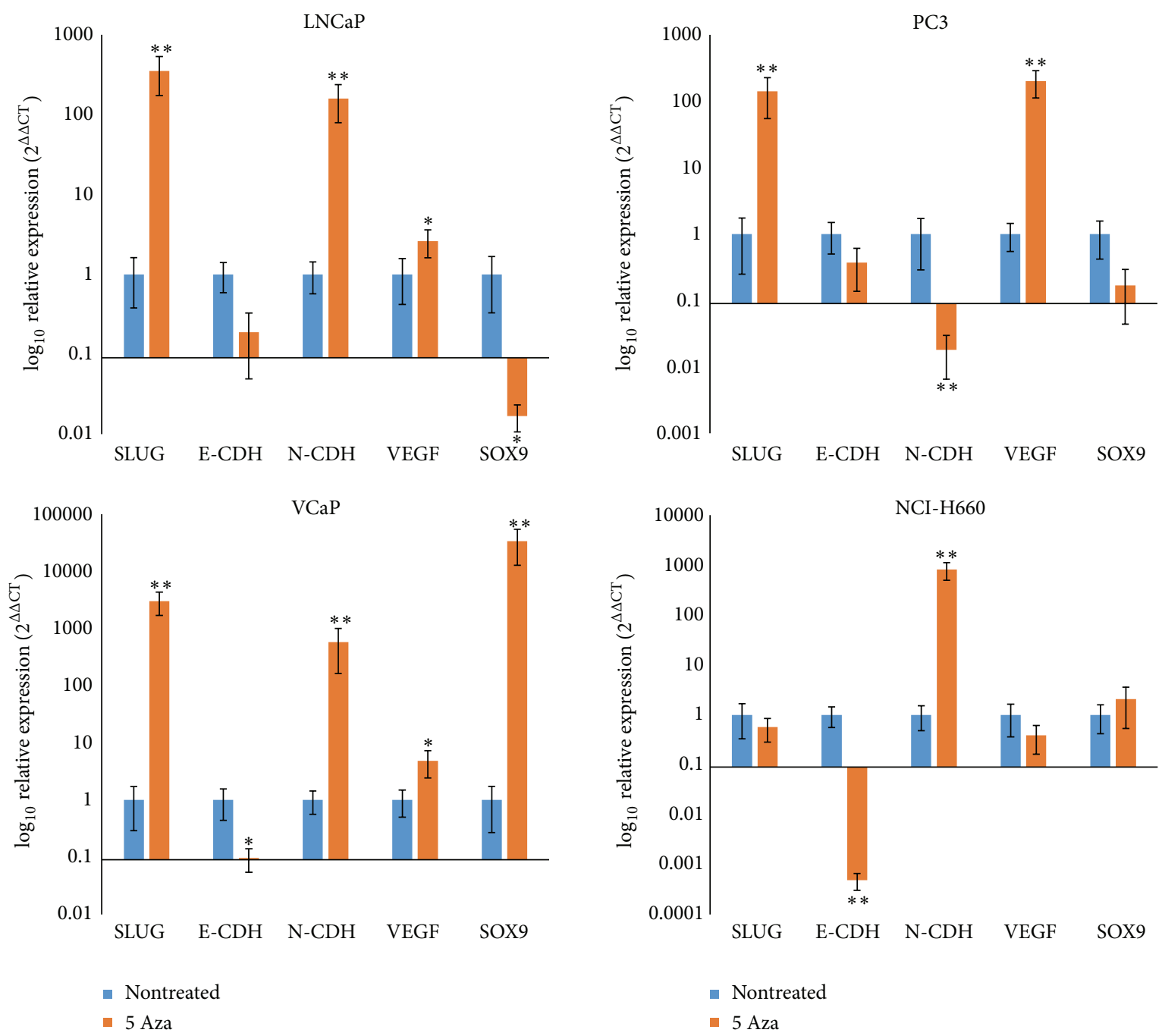

FIGURE 7: Total demethylation suppresses E-cadherin and upregulates N-cadherin and SLUG. 5-AzaC treated or nontreated LNCaP, PC3, VCaP, and NCI-H660 cells were harvested for total RNA. qPCR was performed for SLUG, ECDH, NCDH, VEGFA, SOX9, and the relative gene expression represented as $\log _{10}$ change (Pfaffl, $2^{\Delta \Delta C \mathrm{CT}}$ ), normalized to PGK1, was assessed for LNCaP, PC3, VCaP, and NCI-H660 cell lines. ${ }^{* *} P<0.01,{ }^{*} P<0.05$. Error bars represent s.d., $n=3$ independent biological repeats.

Like data reported in breast cancer models, ETS1 silencing demonstrated that it is a VEGFA positive regulator in prostate cancer cell line model. Runx2 was also reported as a positive VEGF inducer [40], a finding we also confirm in this study. cMYB silencing suggested it has differential role on VEGF induction, being positive regulator only in TMPRSS2ERG fusion-free prostate cancer cell line models (LNCaP, $\mathrm{PC} 3)$. In fusion positive cell lines, $\mathrm{CMYB}$ had a restrictive role (Figures 5 and 6).

3.7. RUNX2 Knockdown Results in SOX9 Downregulation, While ETS1 and cMYB Silencing Result in SOX9 Upregulation in VCaP Cells. Following SOX9 dependency on these transcription factors, we silenced RUNX2 and found it to be a positive SOX9 inducer only in VCaP cell line. In other cell lines it had no significant effect (Figures 5 and 6). RUNX2 and SOX9 were found by Cheng and Genever 2010 [45] to physically interact during mesenchymal stem cell differentiation. SOX9 inhibited the transactivation of RUNX2 and vice versa. The divergence in SOX9 dependence on RUNX2 in other cell lines investigated is most likely related with the different state of global gene network and genome deregulation.

On the contrary, SOX9 dependency on ETS1 was significant in PC3 and VCaP cell lines. Silencing ETS1 abrogated significantly SOX9 expression in PC3 cell line, while in $\mathrm{VCaP}$ cell line, ETS1 silencing resulted in significant SOX9 upregulation (Figures 5 and 6). Gao et al., 2010 [46], found Sox9 upregulated in Ets1-deficient hearts in knockout mouse model, confirming the dependency found by us in VCaP cell line.

cMYB silencing resulted in SOX9 downregulation in PC3 cells, while in VCaP cells cMYB silencing resulted in significant SOX9 upregulation. There was no significant change in SOX9 level upon cMYB silencing in LNCaP and NCI H660 cells. c-Myb transcription factor is associated with 
proliferation of undifferentiated cells in number of tissues, but recent data suggests its role also in differentiation. cMyb is important in formation of the cartilage, bone, and apparently also hard tissue mineralization [47]. Transient transfection of embryonic micromasses using constructs carrying siRNA c-Myb treated cultures expresses significantly lower level of Sox9. The cMyb and Sox9 have probably negative regulatory feedback loop [48].

\subsection{Global Demethylation Reveals SLUG and N-Cadherin} Expression to Be Methylation Dependent. Using 5-azacytidine (5-AzaC), an inhibitor of DNA methyltransferase, we followed the expression of the EMT related genes. We found similar pattern of expression of SLUG, E-cadherin, $\mathrm{N}$-cadherin, and VEGFA in AR preserved signaling cell lines LNCaP and VCaP. SLUG and VEGFA were upregulated in LNCaP, PC3, and VCaP cells. E-Cadherin was downregulated, while $\mathrm{N}$-cadherin was upregulated in LNCaP, VCaP, and NCI H660 cells. SOX9 was downregulated in TMPRSS2-ERG fusion free cell lines (LNCaP, PC3) after 5-AzaC treatment (Figure 7).

\section{Conclusions}

miR-204 gradually loses its tumor-suppressor activities and becomes deregulated in more advanced fusion positive cell lines, promoting EMT in AR dependent and fusion dependent manner. The different models of lymph node and bone marrow metastasis have distinct patterns of expression for various EMT markers and TFs and provide initial means for adapted therapeutic approaches of selective silencing of noncoding RNAs and TFs.

$\begin{array}{ll}\text { Abbreviations } \\ \text { PCa: } & \text { Prostate cancer (carcinoma) } \\ \text { AR: } & \text { Androgen receptor } \\ \text { TF: } & \text { Transcription factor } \\ \text { EMT: } & \text { Epithelial to mesenchymal transition } \\ \text { miR: } & \text { MicroRNA } \\ \text { siRNA: } & \text { Small interfering RNA } \\ \text { RT-qPCR: } & \text { Reverse transcriptase quantitative } \\ & \text { real-time polymerase chain reaction } \\ \text { s.d.: } & \text { Standard deviation } \\ \text { 5-AzaC: } & \text { 5-Aza-cytidine } \\ \text { cMYB: } & \text { v-myb avian myeloblastosis viral } \\ & \text { oncogene homolog } \\ \text { RUNX2: } & \text { runt-related transcription factor } 2 \\ \text { ETS1: } & \text { v-ets avian erythroblastosis virus E26 } \\ & \text { oncogene homolog 1 } \\ \text { ERG: } & \text { v-ets avian erythroblastosis virus E26 } \\ & \text { oncogene homolog } \\ \text { PGK1: } & \text { Phosphoglycerate kinase 1. }\end{array}$

\section{Conflict of Interests}

The authors declare that there is no conflict of interests regarding the publication of this paper.

\section{Authors' Contribution}

Krassimira Todorova and Soren Hayrabedyan designed the study, acquired and analyzed the data, and wrote the paper. Krassimira Todorova did the transfection experiments, qPCR, and FCS. Krassimira Todorova, Diana Zasheva, and Kristiyan Kanev did the cell culturing, cell migration, and CKK-8 assay.

\section{Acknowledgment}

This study was funded by a grant from The National Sciences Fund at Bulgarian Ministry of Education and Sciences, Project: DMU 03/27, provided to Krassimira Todorova, Ph.D. degree.

\section{References}

[1] M. E. Wright, M.-J. Tsai, and R. Aebersold, “Androgen receptor represses the neuroendocrine transdifferentiation process in prostate cancer cells," Molecular Endocrinology, vol. 17, no. 9, pp. $1726-1737,2003$.

[2] Z. Huang, P. J. Hurley, B. W. Simons et al., "Sox9 is required for prostate development and prostate cancer initiation," Oncotarget, vol. 3, no. 6, pp. 651-663, 2012.

[3] S. A. Tomlins, B. Laxman, S. Varambally et al., "Role of the TMPRSS2-ERG gene fusion in prostate cancer," Neoplasia, vol. 10, no. 2, pp. 177-188, 2008.

[4] J. Yu, D. R. Rhodes, S. A. Tomlins et al., "A polycomb repression signature in metastatic prostate cancer predicts cancer outcome," Cancer Research, vol. 67, no. 22, pp. 10657-10663, 2007.

[5] V. Davalos, C. Moutinho, A. Villanueva et al., "Dynamic epigenetic regulation of the microRNA-200 family mediates epithelial and mesenchymal transitions in human tumorigenesis," Oncogene, vol. 31, no. 16, pp. 2062-2074, 2012.

[6] M. M. Shen and C. Abate-Shen, "Molecular genetics of prostate cancer: New prospects for old challenges," Genes and Development, vol. 24, no. 18, pp. 1967-2000, 2010.

[7] C. A. Heinlein and C. Chang, "Androgen receptor in prostate cancer," Endocrine Reviews, vol. 25, no. 2, pp. 276-308, 2004.

[8] C. E. Massie, B. Adryan, N. L. Barbosa-Morais et al., "New androgen receptor genomic targets show an interaction with the ETS1 transcription factor," EMBO Reports, vol. 8, no. 9, pp. 871878, 2007.

[9] Y. Shiozawa, E. A. Pedersen, A. M. Havens et al., "Human prostate cancer metastases target the hematopoietic stem cell niche to establish footholds in mouse bone marrow," Journal of Clinical Investigation, vol. 121, no. 4, pp. 1298-1312, 2011.

[10] N. C. Nicolaides, R. Gualdi, C. Casadevall, L. Manzella, and B. Calabretta, "Positive autoregulation of c-myb expression via Myb binding sites in the $5 /$ flanking region of the human c-myb gene," Molecular and Cellular Biology, vol. 11, no. 12, pp. 61666176, 1991.

[11] F. Kalkbrenner, S. Guehmann, and K. Moelling, "Transcriptional activation by human c-myb and v-myb genes," Oncogene, vol. 5, no. 5, pp. 657-661, 1990.

[12] M. Lagos-Quintana, R. Rauhut, A. Yalcin, J. Meyer, W. Lendeckel, and T. Tuschl, "Identification of tissue-specific MicroRNAs from mouse," Current Biology, vol. 12, no. 9, pp. 735-739, 2002. 
[13] J. Lin, Q. Cao, J. Zhang et al., "MicroRNA expression patterns in indeterminate inflammatory bowel disease," Modern Pathology, vol. 26, no. 1, pp. 148-154, 2013.

[14] R. Narayanan, J. Jiang, Y. Gusev et al., "Micrornas are mediators of androgen action in prostate and muscle," PLOS ONE, vol. 5, no. 10, Article ID e13637, 2010.

[15] M. Karaivanov, K. Todorova, A. Kuzmanov, and S. Hayrabedyan, "Quantitative immunohistochemical detection of the molecular expression patterns in proliferative inflammatory atrophy," Journal of Molecular Histology, vol. 38, no. 1, pp. 1-11, 2007.

[16] K. Todorova, S. Zoubak, M. Mincheff, and S. Kyurkchiev, "Biochemical nature and mapping of PSMA epitopes recognized by human antibodies induced after immunization with gene-based vaccines," Anticancer Research, vol. 25, no. 6, pp. 4727-4732, 2005.

[17] K. Todorova, I. Ignatova, S. Tchakarov et al., "Humoral immune response in prostate cancer patients after immunization with gene-based vaccines that encode for a protein that is proteasomally degraded," Cancer Immunity, vol. 5, article 1, 2005.

[18] K. Todorova, M. Mincheff, S. Hayrabedyan et al., "Fundamental role of microRNAs in androgen-dependent male reproductive biology and prostate cancerogenesis," American Journal of Reproductive Immunology, vol. 69, no. 2, pp. 100-104, 2013.

[19] M. Korpal, E. S. Lee, G. Hu, and Y. Kang, "The miR-200 family inhibits epithelial-mesenchymal transition and cancer cell migration by direct targeting of E-cadherin transcriptional repressors ZEB1 and ZEB2," The Journal of Biological Chemistry, vol. 283, no. 22, pp. 14910-14914, 2008.

[20] J. Huang, L. Zhao, L. Xing, and D. Chen, "MicroRNA-204 regulates Runx2 protein expression and mesenchymal progenitor cell differentiation," Stem Cells, vol. 28, no. 2, pp. 357-364, 2010.

[21] Z. Gibas, R. Becher, E. Kawinski, J. Horoszewicz, and A. A. Sandberg, "A high-resolution study of chromosome changes in a human prostatic carcinoma cell line (LNCaP)," Cancer Genetics and Cytogenetics, vol. 11, no. 4, pp. 399-404, 1984.

[22] M. E. Kaighn, K. S. Narayan, Y. Ohnuki, J. F. Lechner, and L. W. Jones, "Establishment and characterization of a human prostatic carcinoma cell line (PC-3)," Investigative Urology, vol. 17, no. 1, pp. 16-23, 1979.

[23] S. Korenchuk, J. E. Lehr, L. McLean et al., "VCaP, a cell-based model system of human prostate cancer," In Vivo, vol. 15, no. 2, pp. 163-168, 2001.

[24] S.-L. Lai, H. Brauch, T. Knutsen et al., "Molecular genetic characterization of neuroendocrine lung cancer cell lines," Anticancer Research, vol. 15, no. 2, pp. 225-232, 1995.

[25] M. Ishiyama, H. Tominaga, M. Shiga, K. Sasamoto, Y. Ohkura, and $\mathrm{K}$. Ueno, "A combined assay of cell viability and in vitro cytotoxicity with a highly water-soluble tetrazolium salt, neutral red and crystal violet," Biological and Pharmaceutical Bulletin, vol. 19, no. 11, pp. 1518-1520, 1996.

[26] M. A. Markus, R. B. Gerstner, D. E. Draper, and D. A. Torchia, "Refining the overall structure and subdomain orientation of ribosomal protein $S 4 \Delta 41$ with dipolar couplings measured by NMR in uniaxial liquid crystalline phases," Journal of Molecular Biology, vol. 292, no. 2, pp. 375-387, 1999.

[27] P. O. Krutzik and G. P. Nolan, "Intracellular phospho-protein staining techniques for flow cytometry: monitoring single cell signaling events," Cytometry A, vol. 55, no. 2, pp. 61-70, 2003.

[28] J. M. Irish, R. Hovland, P. O. Krutzik et al., "Single cell profiling of potentiated phospho-protein networks in cancer cells," Cell, vol. 118, no. 2, pp. 217-228, 2004.
[29] Y. Sun, S. Koo, N. White et al., "Development of a micro-array to detect human and mouse microRNAs and characterization of expression in human organs," Nucleic Acids Research, vol. 32, no. 22, p. e188, 2004.

[30] D. P. Turner, V. J. Findlay, O. Moussa et al., "Mechanisms and functional consequences of PDEF protein expression loss during prostate cancer progression," Prostate, vol. 71, no. 16, pp. 1723-1735, 2011.

[31] J. S. Imam, J. R. Plyler, H. Bansal et al., "Genomic loss of tumor suppressor miRNA-204 promotes cancer cell migration and invasion by activating $\mathrm{AKT} / \mathrm{mTOR} / \mathrm{Racl}$ signaling and actin reorganization," PLoS ONE, vol. 7, no. 12, Article ID e52397, 2012.

[32] Y. Lee, X. Yang, Y. Huang et al., "Network modeling identifies molecular functions targeted by miR-204 to suppress head and neck tumor metastasis," PLoS Computational Biology, vol. 6, no. 4, Article ID e1000730, 2010.

[33] S. Sharma and A. Lichtenstein, "Aberrant splicing of the Ecadherin transcript is a novel mechanism of gene silencing in chronic lymphocytic leukemia cells," Blood, vol. 114, no. 19, pp. 4179-4185, 2009.

[34] I. M. Shapiro, A. W. Cheng, N. C. Flytzanis et al., "An EMTdriven alternative splicing program occurs in human breast cancer and modulates cellular phenotype," PLoS Genetics, vol. 7, no. 8, Article ID e1002218, 2011.

[35] C. Cai, H. Wang, H. H. He et al., "ERG induces androgen receptor-mediated regulation of SOX9 in prostate cancer," Journal of Clinical Investigation, vol. 123, no. 3, pp. 1109-1122, 2013.

[36] H.-F. Yuen, W.-K. Kwok, K.-K. Chan et al., “TWIST modulates prostate cancer cell-mediated bone cell activity and is upregulated by osteogenic induction," Carcinogenesis, vol. 29, no. 8, pp. 1509-1518, 2008.

[37] J. Gavard, V. Patel, and J. S. Gutkind, "Gutkind, Angiopoietin-1 prevents VEGF-induced endothelial permeability by sequestering Src through mDia," Developmental Cell, vol. 14, no. 1, pp. 25-36, 2008.

[38] A. Courboulin, R. Paulin, N. J. Giguère et al., "Role for miR204 in human pulmonary arterial hypertension," Journal of Experimental Medicine, vol. 208, no. 3, pp. 535-548, 2011.

[39] E. Lambertini, G. Lisignoli, E. Torreggiani et al., "Slug gene expression supports human osteoblast maturation," Cellular and Molecular Life Sciences, vol. 66, no. 22, pp. 3641-3653, 2009.

[40] J. Akech, J. J. Wixted, K. Bedard et al., "Runx2 association with progression of prostate cancer in patients: mechanisms mediating bone osteolysis and osteoblastic metastatic lesions," Oncogene, vol. 29, no. 6, pp. 811-821, 2010.

[41] S. K. Baniwal, O. Khalid, Y. Gabet et al., "Runx2 transcriptome of prostate cancer cells: insights into invasiveness and bone metastasis," Molecular Cancer, vol. 9, article 258, 2010.

[42] E. Lambertini, T. Franceschetti, E. Torreggiani et al., "SLUG: a new target of lymphoid enhancer factor-1 in human osteoblasts," BMC Molecular Biology, vol. 11, article 13, 2010.

[43] G. H. Little, S. K. Baniwal, H. Adisetiyo et al., "Differential effects of RUNX2 on the androgen receptor in prostate cancer: synergistic stimulation of a gene set exemplified by SNAI2 and subsequent invasiveness," Cancer Research, vol. 74, no. 10, pp. 2857-2868, 2014.

[44] T. Shirakihara, M. Saitoh, and K. Miyazono, "Differential regulation of epithelial and mesenchymal markers by $\delta \mathrm{EF} 1$ proteins in epithelial-mesenchymal transition induced by TGF$\beta$," Molecular Biology of the Cell, vol. 18, no. 9, pp. 3533-3544, 2007. 
[45] A. Cheng and P. G. Genever, "SOX9 determines RUNX2 transactivity by directing intracellular degradation," Journal of Bone and Mineral Research, vol. 25, no. 12, pp. 2680-2689, 2010.

[46] Z. Gao, G. H. Kim, A. C. Mackinnon et al., "Ets1 is required for proper migration and differentiation of the cardiac neural crest," Development, vol. 137, no. 9, pp. 1543-1551, 2010.

[47] E. Matalová, M. Buchtová, A. S. Tucker et al., "Expression and characterization of c-Myb in prenatal odontogenesis," Development Growth and Differentiation, vol. 53, no. 6, pp. 793803, 2011.

[48] V. Oralova, M. Buchtova, E. Janeckova, A. Tucker, and E. Matalova, "Modulation of c-Myb during chondrogenesis," Bone Abstracts, vol. 1, Article ID PP252, 2013. 


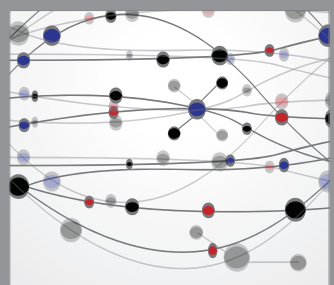

The Scientific World Journal
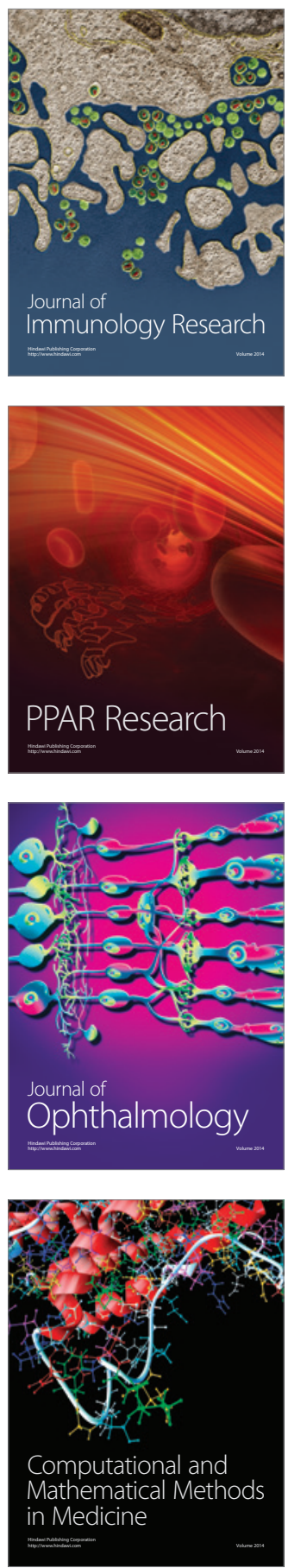

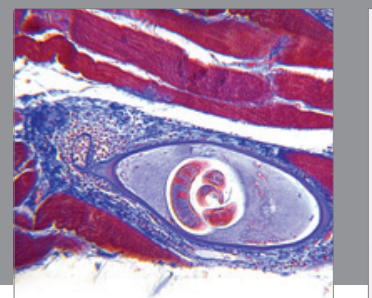

Gastroenterology

Research and Practice
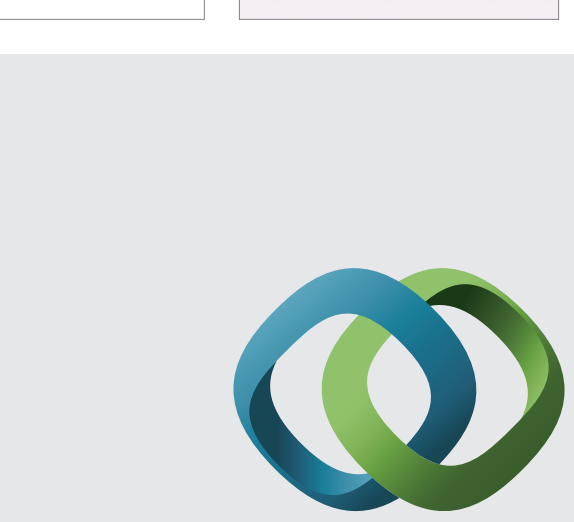

\section{Hindawi}

Submit your manuscripts at

http://www.hindawi.com
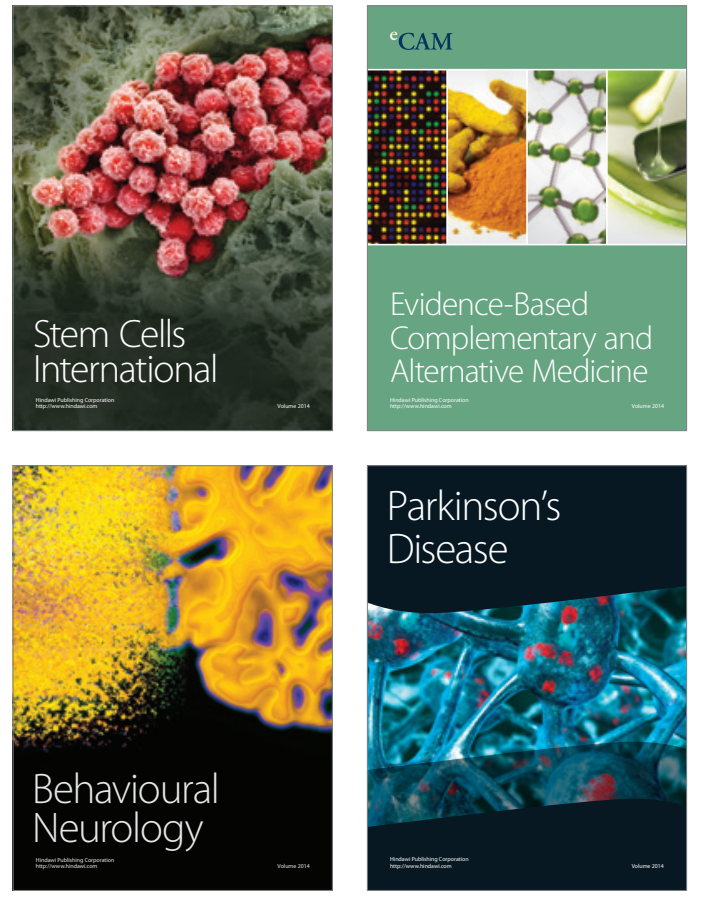
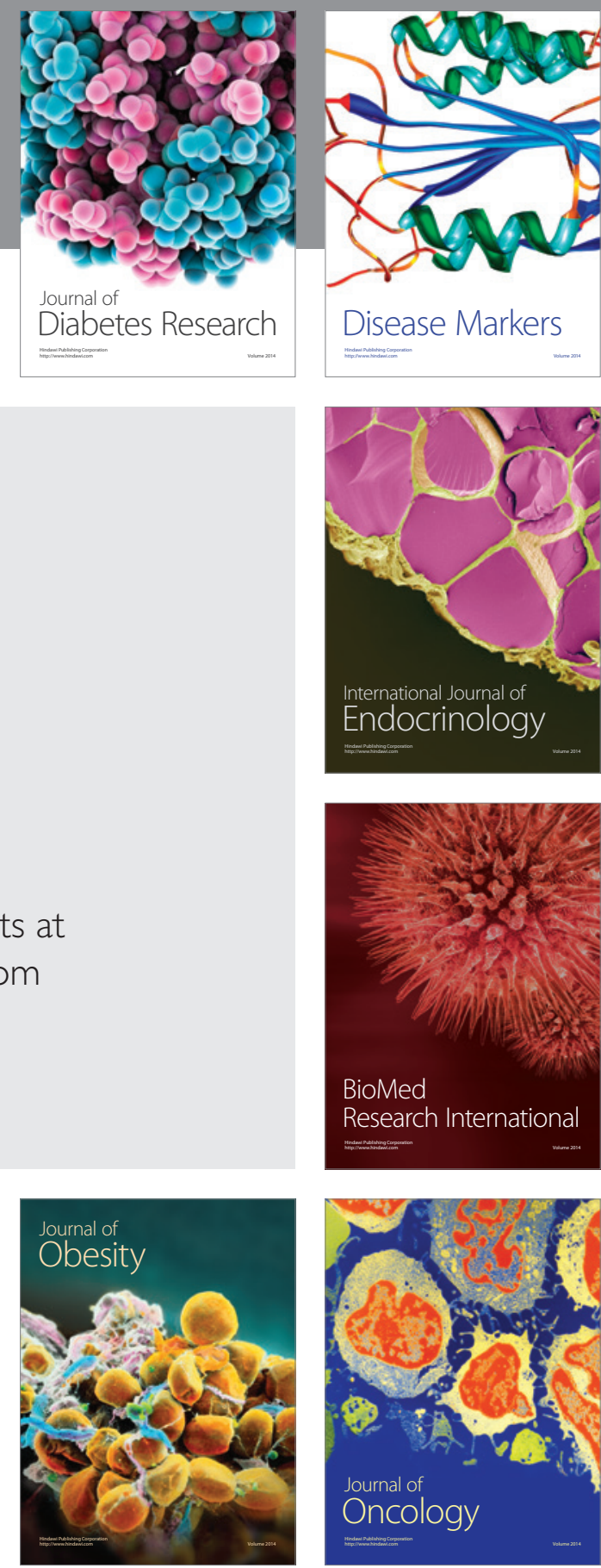

Disease Markers
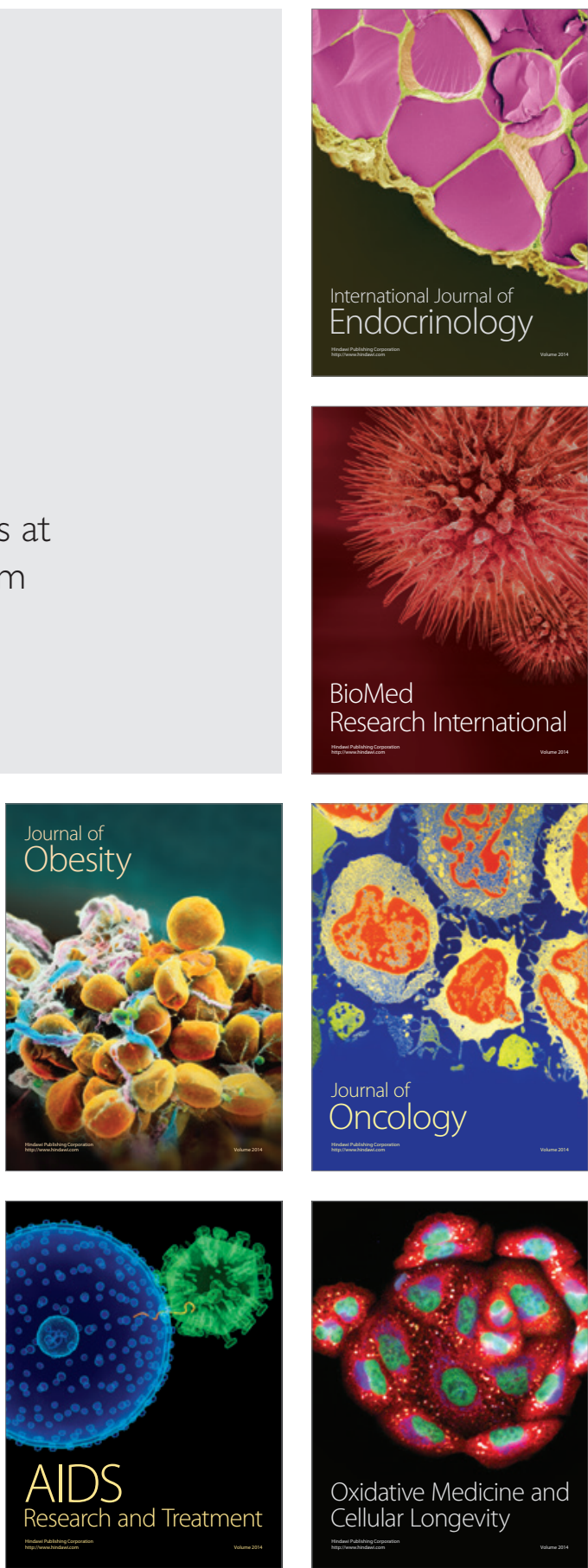